\title{
The effect of defect size and soil aggressivity on corrosion of underground oil \& gas pipelines
}

\author{
Maros Halama ${ }^{1, *}$, Emily Haluschak ${ }^{2}$, Peter Hanzes ${ }^{1}$ and Gabriela Baranova ${ }^{1}$ \\ ${ }^{1}$ Technical University of Kosice, Faculty of Materials, Metallurgy and Recycling, Letna 9, 042 00, Kosice, Slovakia \\ ${ }^{2}$ Purdue University, Davidson School of Chemical Engineering, West Lafayette, 47907 Indiana, USA
}

\begin{abstract}
Nowadays, the oil \& gas industry has limited number of non-destructive corrosion techniques for assessment of life-time of existing infrastructures, especially on low and medium pressure pipelines exposed to aggressive soil environment where defect in isolation and presence of water formed ideal conditions for corrosion attack. Efficient non-destructive corrosion monitoring can be achieved using mobile potentiostat through the selection of appropriate monitoring techniques and special kind of sensor. OCP potential is monitored for 10 minutes and actual corrosion rate of pipeline with corrosion products in soil mixture was determined using Stern polarization technique in non-invasive arrangement. Using global database with range of minimum and maximum corrosion rate limits obtained from terrain measurements we can determine active or passive role of corrosion products and assess risk joint with aggressiveness of soil in location of interest. Finally, values were divided between high active, medium active and passive corrosion. Output is alarm, which can help in decision if reconstruction of underground pipelines where isolation failed in time is sufficient or not. When done properly, corrosion monitoring using non-invasive "in-situ" technique can serve as an early warning system before the onset of corrosion related failures.
\end{abstract}

\section{Introduction}

Corrosion is in general dangerous phenomenon in today's industrial world. Annual damage, which is attributed to corrosion worldwide, is app. $3 \%$ of global GDP. For each gas distribution system operator, corrosion poses a serious threat to the integrity of the assets being operated. Indeed, the value of these assets is generated or maintained only when they are able to fulfill their primary function, which is the safe and reliable distribution of natural gas to final customers. The limited financial resources that operators are able to earmark for reparation of corrosion damage just underline the importance of this problem. Corrosion of buried pipelines due to the failures in isolation is of high importance in oil and gas industry. Techniques for monitoring corrosion and related parameters in field applications have been reported by NACE Technical Committee in 2012 followed by revised ASTM G96 Standard guide for online monitoring of corrosion in plant equipment (Electrical and electrochemical methods) in 2014 and ASTM G59 Standard test method for conducting potentiodynamic polarization resistance measurements was also revised in 2014. Nowadays trends in simulation and prediction techniques [1] and non-destructive techniques suitable for terrain measurements [2,3] have created new tasks in wide range of industry, not only oil and gas. In the field of inspection important advances have been made in miniaturisation of equipment and processing of data. For corrosion monitoring these include measuring of OCP of pipeline or measuring of polarisation resistance on surface with detecting activity of corrosion products in specific location. So, having such a embedded sensor based on "in-situ" measurements directly on pipeline surface could serve as decision tool for risk assessment of pipelines.

For this reason there is a strong sector-wide demand for an optimal strategy that will deliver targeted corrosion monitoring, risk-based structured data collection and evaluation, and reliable recommendations for correct interventions. Such an approach will allow the operator to allocate scarce resources effectively. Thanks for support of SPP - distribution, a.s., we have checked adaption of this ambition during last 3 years and decide to shift qualitatively the corrosion monitoring in terrain. It allows more targeted identification of hazardous parts of local networks and thus leads to a more accurate allocation of dedicated resources. Due to the electrochemical principle of corrosive attack on the pipeline, effective corrosion monitoring based on the measurement of polarization resistance on the surface of the pipe. This approach provides relatively accurate information on the corrosion rate of steel and the anticorrosion protection of layers. For any oil \& gas industry, performance of these measurements is extremely interesting through the use of mobile potentiostats and sensors that provide de facto laboratory results in the field.

\footnotetext{
* Corresponding author: maros.halama $@$ tuke.sk
} 


\subsection{Implementation in terrain}

In the first step, it was necessary to provide a mobile potentiostat for the performance of the field measurements, which fulfilled practical requirements such as electrical self-sufficiency (powered by Li-ion battery), resistance under atmospheric conditions and performance that would achieve the parameters of laboratory instruments. The successful verification of whether this measurement, which yields to identical results as destructive laboratory techniques after extraction and collection of samples from terrain, allow us to do further testing and implementation. The next step was development sensor, which would allow fixation on pipelines of different dimensions and filling the electrolyte in different positions (Fig. 1). Measurements were realized using mixture of soil and water as electrolyte simulated the environment in which the monitored pipeline is located.

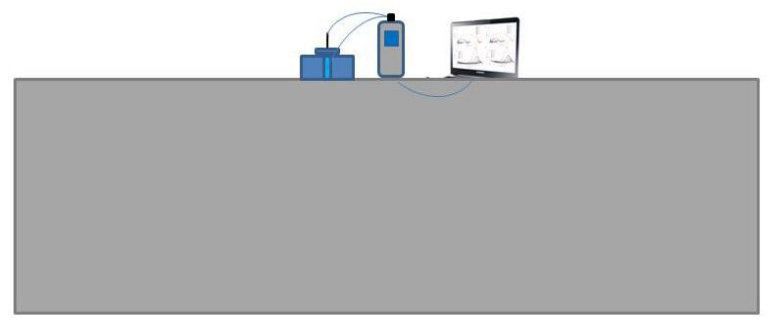

Fig. 1. Non-invasive corrosion monitoring of underground pipelines.

\section{Experimental}

\subsection{OCP monitoring}

The field measurements were carried out in several maintenance areas of local networks with planned zones for reconstruction, while the NTL gas pipelines with relatively high age and low leakage, which is a natural phenomenon in NTL network operation, represented the target group of gas pipelines. The aim was to verify their real technical condition, respectively to determine their residual life-time. When performing potentiometric measurements, the status of activity/passivity of corrosion products on the steel surface could be determined from the measurement of OCP potentials (free corrosion potential), and based on this measurement, their protective character can be determined on the basis of so-called Pourbaix criteria. Pourbaix criterion expresses the electrochemical balance of metal or solid undergo corrosion via interaction with the aqueous environment. OCP measurements were realized during first $10 \mathrm{~min}$ of application of sensor on the surface of pipeline. OCP map on Fig.2 represented preliminary determination of potential in surrounding of perforation caused by massive corrosion attack. Minimum OCP potential of less than $-340 \mathrm{mV}$ vs. SCE was detected close to perforation, which means indication of active sites with no protection. On the other hand, potential around $-100 \mathrm{mV}$ vs. SCE showed partly protected sites by corrosion products.

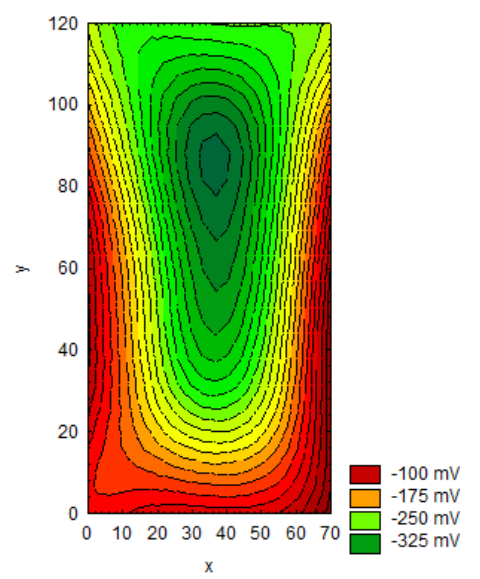

Fig. 2. OCP mapping in surrounding of corrosion perforation on pipeline.

\subsection{Effect of size of defect on corrosion}

We have used the polarization resistance measurement technique for relatively accurate and rapid verification of the actual corrosion rate, which also fulfills the nondestructive monitoring criterion.

At first we have checked effect of size of defect on actual corrosion rate in laboratory conditions (see Fig.3). It seems that diameter smaller than $4 \mathrm{~mm}$ is characterized mainly by dramatic way of degradation due to the concentration of all reactions in small area and preferable mechanism is pitting.

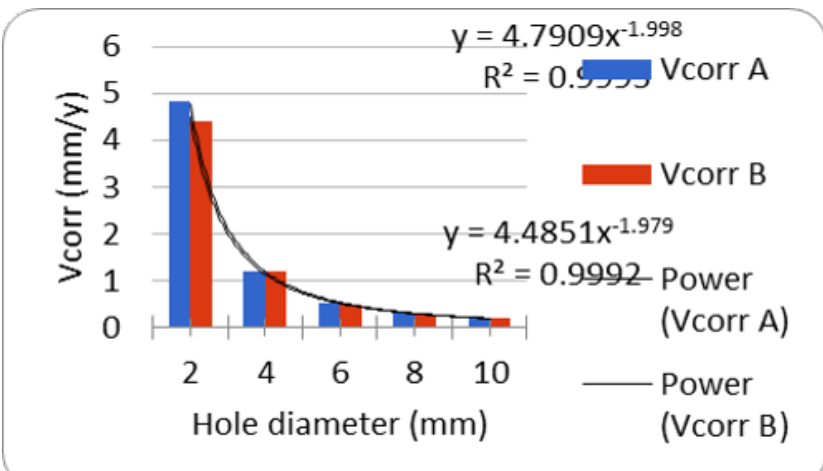

Fig. 3. Actual corrosion rate vs. size of defect on steel pipeline.

\subsection{Effect of aggressivity of soil on corrosion}

When examining the aggressiveness of the surrounding soil on the corrosion rate of the pipeline, measurements were made on the polished surface of the steel grade for gas industry X60. Measurements have shown that this technique can somewhat estimate the behavior of steel after failure in insulation in a given location.

Sensor was used for "in-situ" polarisation measurements on pipeline with both ground finished 
surface and with corrosion products in two different types of soil mixtures with $\mathrm{pH} 8.8$ and 7.9. Due to the double activity of corrosion in soil mixture with $\mathrm{pH} 8.8$ (it has higher conductivity and total dissolved solids) vs. soil mixture with $\mathrm{pH} 7.7$ and difference between fresh iron surface and surface with corrosion products, sensor is able to detect between high activity, middle activity and passivity (see Fi. 3a,b).

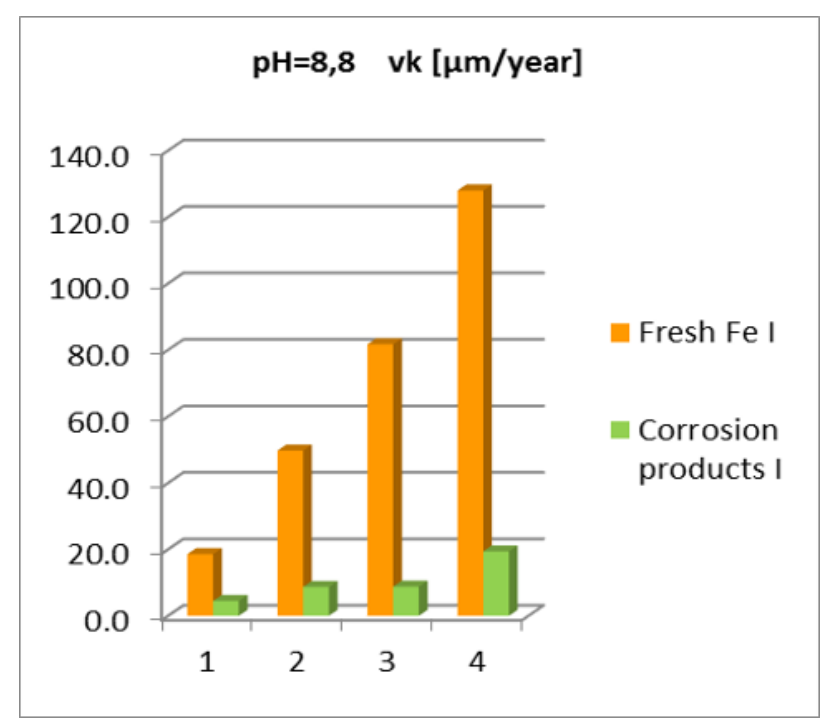

Fig. 3a. In-situ polarisation measurements on pipeline by Noninvasive corrosion monitoring technique using sensor in terrain, soil mixture of $\mathrm{pH}=8.8$.

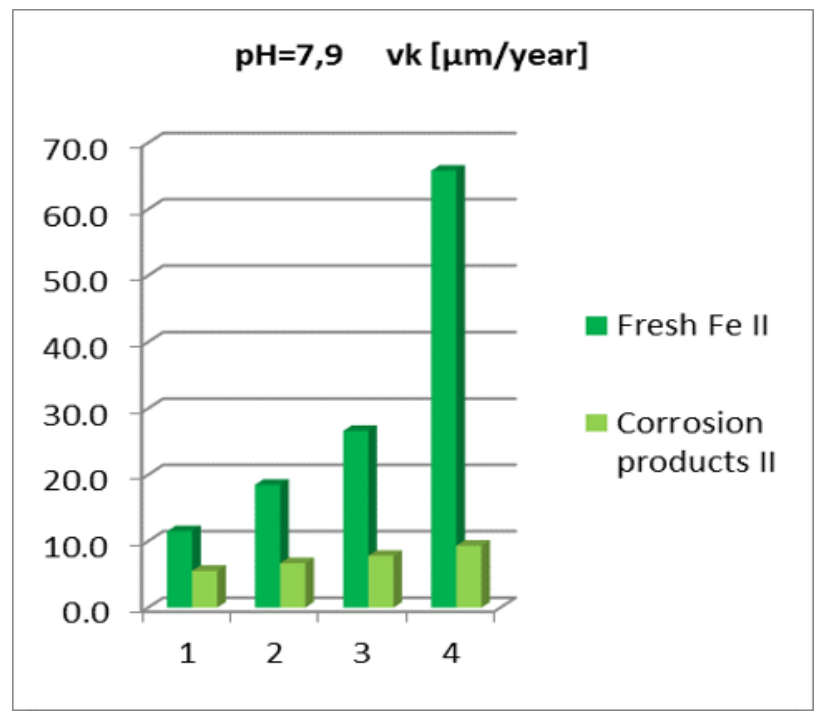

Fig. 3b. In-situ polarisation measurements on pipeline by Noninvasive corrosion monitoring technique using sensor in terrain, soil mixture of $\mathrm{pH}=7.9$.

Based on the experience of more than 300 measurements in laboratory conditions and field measurements, a traffic light alarm was set for the values of the actual corrosion rates detected on the ground surface of the pipeline, which determined the range of values for each level of corrosion - negligible (safe zone), not negligible (slightly dangerous zone) and high corrosive activity (hazardous zone). In a similar way, a traffic light alarm was set also for the values of actual corrosion rates detected for measuring points with corrosion products in soil extracts. From these measurements it is possible to conclude what the role of the protective function of corrosion products depend on soil conditions at studied location. An interesting finding was the measurement in the area of SW country, where there are sites with a very suitable geological composition of the soil, where the lowest corrosion rates were measured at all (around $2.5 \mu$ m.year-1). Under such sand bed conditions, the 50-year old pipelines showed no corrosion attack. In the given location, the measurement helped to optimize the extent of the reconstruction, from which the gas pipelines with the reached age criterion were but in excellent technical condition without corrosion. In the case of perforation of a pipeline or a corrosion accident, it is mostly a concentrated local influence of aggressive components of the environment at which higher corrosion rates are achieved.

To take into account these conditions, we proceeded to measure also in an artificial soil electrolyte that has a higher conductivity and contains a higher amount of dissolved salts. Based on the experience of field measurements, a similarly set alarm for the values of the actual corrosion rates was determined by the ranges of values in the category of negligible, non-negligible and high corrosion rates.

\section{Conclusions}

The proposed non-invasive technique for corrosion monitoring using miniaturized sensor used for "in-situ" measurements on pipeline could be useful corrosion manager tool/step after DCVG technique. Developed sensor is able to detect role of protection character of corrosion products together with actual corrosion rate on pipeline at location of interest. It was observed that it has enough sensitivity to detect between high corrosion risk on active corrosion sites; middle corrosion, where corrosion products play partly protection function and "zero" level corrosion sites. This work served for scientific verification of risk assessment model for pipeline networks using alarm criterion. Therefore, for complex evaluation of service-life of pipeline should be taken into account also other properties such as mechanical parameters, fatigue etc.

An added value of non-destructive corrosion monitoring based on potentiometric measurements is the information on the current state of corrosion activity on distribution network pipelines after multi-year operation. It is also possible to determine the role of environmental aggressivity on the steel piping, while the simulation of extreme conditions can also be used to estimate the service life of the pipelines in the localities. We see this approach has potential to be a new, complementary tool (decision-making criterion) for the strategy of future operations, in particular for NTL pipelines of local networks, which we are able to analyze in a more detailed way about their technical condition and estimated service-life expectancy. The current challenge is to ensure a structured data collection from corrosion 
monitoring and the creation of a robust corrosion database that will contain permissible corrosion parameters for steel pipelines of local networks. In doing so, account will be taken of local conditions such as e.g. the aggressiveness of the soil and with the increasing experience of field measurements, the adjustment of the sensitivity of the functions of the so-called alarm. The output can then be the prediction of the life-time of the pipelines of local networks, while in the long-term and high-quality collection of input data we can consider in the future a more sophisticated approach such as method of artificial neural networks.

\section{References}

1. Hedges, B., Knox, T., Papavinasam, S., Sprague, T.: Monitoring and inspection techniques for corrosion in oil and gas production, In: Corrosion 2015, NACE International Corrosion Conference Series, USA, 2015

2. Papavinasam, S. Doiron, A., Revie, R.W.: Industry survey on techniques to monitor internal corrosion, In: Materials Performance, 51 (2), pp. 34-38

3. Halama, M., Kreislova, K., Lysebettens, J. V.: Prediction of Atmospheric Corrosion of Carbon Steel Using Artificial Neural Network Model in Local Geographical Regions, In: Corrosion: The Journal of Science and Engineering, Vol. 67, no. 6 (2011), p. 16 , ISSN 0010-9312

\section{Acknowledgements}

The authors gratefully acknowledge SPP-distribution a.s. for support of research project and partially Slovak Research and Development Agency under the project APVV-16-0359 and work was partly realized also within the frame of the Operational Program ERDF: "Research Center of advanced materials and technologies for current and future applications - PROMATECH", project code ITMS: 26220220186. 